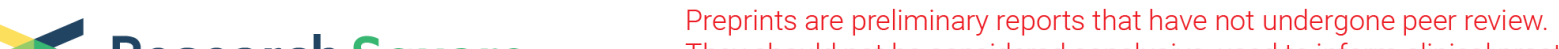 Research Square They should not be considered conclusive, used to inform clinical practice, or referenced by the media as validated information.
}

\section{High CENPA expression in papillary renal cell carcinoma tissues is associated with poor prognosis}

Junwu li

the Second Affiliated Hospital of Chongqing Medical University

Qinke Li

the Second Affiliated Hospital of Chongqing Medical University

Yiteng Xie

the Second Affiliated Hospital of Chongqing Medical University

Yuanfeng Zhang

the Second Affiliated Hospital of Chongqing Medical University

Ronggui Zhang ( $\nabla$ 300452@hospital.cqmu.edu.cn )

the Second Affiliated Hospital of Chongqing Medical University

\section{Research Article}

Keywords: CENPA, Papillary renal cell carcinoma, Prognostic analysis, Bioinformatics

Posted Date: February 24th, 2022

DOI: https://doi.org/10.21203/rs.3.rs-1371578/v1

License: (c) (1) This work is licensed under a Creative Commons Attribution 4.0 International License. Read Full License 


\section{Abstract}

Objective: This work focused on investigating the relation of centromeric protein A (CENPA) gene expression with prognosis of papillary renal cell carcinoma (PRCC).

Methods: We obtained data from PRCC cases in TCGA. Thereafter, CENPA levels between the paired PRCC and matched non-carcinoma samples were analyzed by Wilcoxon rank-and-sum test, while the relations of clinicopathological characteristics with CENPA level were examined by logistic regression and Wilcoxon rank-sum test. The prognostic value of CENPA was assessed by plotting the receiver operating feature curve (ROC) and calculating the value of area under curve (AUC). In addition, relations between clinicopathological features and PRCC survival were analyzed through Kaplan-Meier (KM) and Cox regression analyses. Additionally, we constructed a nomogram according to multivariate Cox regression results for predicting how CENPA affected patient survival. We also determined CENPA levels within cancer and matched non-carcinoma samples through immunohistochemistry (IHC). Finally, we utilized functional enrichment for identifying key pathways related to differentially expressed genes (DEGs) between PRCC cases with CENPA up-regulation and down-regulation.

Results: CENPA expression enhanced in PRCC tissues compared with healthy counterparts $(P<0.001)$. CENPA up-regulation was related to clinical stage and pathological TNM stage $(P<0.05)$. Meanwhile, the ROC curve indicated that CENPA had a remarkable diagnostic capacity for PRCC (AUC=0.936). As revealed by KM curve, PRCC cases with CENPA up-regulation were associated with poor survival compared with those with CENPA down-regulation (risk ratio, $\mathrm{RR}=3.07,95 \% \mathrm{Cl}: 1.58-5.97, \mathrm{P}=0.001$ ). In the meantime, univariate as well as multivariate analysis showed an independent association of CENPA with overall survival (OS, $P<0.001)$. IHC analysis indicated that PRCC cases showed an increased CENPA positive rate compared with controls. As revealed by functional annotations, CENPA was enriched into pathways associated with KEGG - NEUROACTIVE - LIGAND - RECEPTOR - INTERACTION, KEGG CYTOKINE - CYTOKINE - RECEPTOR INTERACTION, NABA - ECM - REGULATORS, NABA - ECM GLYCOPROTEINS, and NABA - CORE - MATRISOME pathways.

Conclusion: CENPA expression increases within PRCC samples, which predicts dismal PRCC survival. CENPA may become a molecular prognostic marker and therapeutic target for PRCC patients.

\section{Introduction}

Papillary renal cell carcinoma (PRCC) is regarded as the second most common subtype of renal cancer, accounting for approximately $15 \%-20 \%$ of the total renal cancer cases ${ }^{[1]}$. The underlying molecular mechanisms of PRCC, a heterogeneous disease, are not completely understood yet ${ }^{[2]}$. Meanwhile, the efficacy of targeted therapy in patients with advanced PRCC remains uncertain, although many recent studies are being conducted to explore the best risk gene characteristics in PRCC. As a result, it is of crucial importance to identify new prognostic biomarkers and therapeutic targets to improve the survival rate of PRCC patients. 
Centromeric protein A (also known as centromere protein A, CENPA) is a variant of the centromerespecific histone $\mathrm{H} 3$, which has a key role in centromere formation. It can transform the centromere into a complex consisting of a chromosome and a protein while ensuring the normal formation and function of the centromere and kinetomeres. In terms of cell-cycle regulation, cell division, and gene stability, the elevated CENPA gene expression can lead to CENPA mislocalization. Subsequently, the ectopic new centromeres and kinetomeres are formed in the chromosomal arms. These ectopic structures can then disrupt the normal segregation of chromosomes in cell division and aneuploidy formation, resulting in tumorigenesis. Alternatively, there is a direct link between the elevated CENPA gene expression and genomic instability, a condition that also triggers cancer occurrence and promotes disease progression. Numerous studies have identified the aberrant expression of CENPA gene in cancers, but related data in PRCC have not been reported yet.

The present work focused on illustrating the relation of CENPA with PRCC and analyzing CENPA's effect on predicting PRCC prognosis according to TCGA-derived RNA-sequencing (RNA-seq) data. Besides, CENPA expression within PRCC as well as matched samples was analyzed, then, the relation of CENPA expression with overall survival (OS) of patients was analyzed. In addition, prognostic and clinical relevance studies were conducted for exploring the significance of CENPA in diagnosing and predicting patient prognosis. Moreover, enrichment, immune infiltration correlation, together with molecular interaction network analyses were carried out to check the biological value of CENPA. Collectively, CENPA is an important and independent predictor of PRCC.

\section{Materials And Methods}

\subsection{RNA-seq data sources}

Totally 321 PRCC cases with available gene expression data (KIRP-TPM) were gathered from TCGA database, while samples with RNA-seq data but without corresponding clinical data were excluded. Meanwhile, TPM data from 321 PRCC patients were further analyzed after filtering, and gene expression data were classified into low and high expression groups on the basis of the median CENPA expression level. This study complied with the publication guidelines formulated by the TCGA, and all the data used in this work were obtained from TCGA, so the patient ethical approval and informed consent were not needed.

\subsection{Enrichment analysis}

The Interacting Gene Retrieval Tool (STRING) online database was applied in predicting protein-protein interaction (PPI) network for CENPA co-expression genes and examining the functional interactions of proteins. Typically, we determined interactions whose comprehensive scores were over 0.4 to be of statistical significance. In addition, we compared gene levels (HTSeq-Counts) in CENPA up-regulation and down-regulation groups. Thereafter, by adopting Wilcoxon rank-sum test of DESeq2 software (3.8), we identified the differentially expressed genes (DEGs) between CENPA up-regulation and down-regulation 
groups on this basis. This study selected adjusted $\mathrm{P}<0.05$ and |log Fold change (FC) $\mid>1.5$ to be the thresholds to identify DEGs. Those identified DEGs were later subject to Gene Ontology (GO) analysis with Clusterprofiler software 3.6.0.

\subsection{Gene enrichment analysis}

This study adopted GSEA for determining the statistical significance of a gene set and the uniform difference between 2 biological statuses. We conducted GSEA with ClusterProfiler in R package for illustrating the significant differences in functions and pathways between CENPA up-regulation and down-regulation groups. Functions and pathways satisfying the thresholds of false discovery rate (FDR) $<0.25$ and adjusted $\mathrm{P}<0.05$ were deemed to be significantly enriched.

\subsection{Immune infiltration analysis was performed by single sample of GSEA (sSGSEA)}

CENPA samples were analyzed by ssGSEA with R software GSVA package. Twenty-four immune cell types were analyzed, including mast cells, neutrophils, macrophages, eosinophils, CD56bright NK cells, CD56dim NK cells, natural killer (NK) cells, activated DC (aDC), dendritic cells (DC), immature DC (iDC), plasmacyte-like DC (pDC), CD8 + T cells, T cells, Th1/Th2/Th17 cells, T helper (Th) cells, regulatory $T$ cells (Treg), T follicle helper cells, effector memory T cells (Tem), central memory T cells (Tcm), B cells, cytotoxic cells, and T cells (Tgd). We determined the enrichment score of every cell type based on gene levels within cancer samples and those reported key genes in 24 types of immune cells. Additionally, we conducted Spearman correlation for determining the relation of PRCC with immune cell subpopulations. In addition, Wilcoxon was adopted for investigating the immune cell infiltration levels in CENPA upregulation and down-regulation groups by the rank-sum test.

\subsection{Construction and evaluation of the nomograms}

We established nomograms by R software RMS package according to multivariate results to predict 1-, 3and 5-year survival of different individuals, which include clinicopathological features and calibration maps that were significantly associated with CENPA. Calibration curve is the most commonly used method to evaluate model performance, which was assessed graphically through comparing our nomogram-estimated values with actual results in the present work, and $45^{\circ}$-line represented the optimal predicted results. Moreover, we also adopted concordance index (C-index) for assessing our nomogram discrimination. Then, we compared the prediction accuracy between prognostic factors and our nomograms based on ROC analysis and C-indexs, and P < 0.05 stood for statistical significance.

\subsection{Statistical analysis}

R (version 3.6.3) software was employed for performing statistical analysis. CENPA levels within tumor and non-carcinoma samples were explored by Wilcoxon rank-sum test. Besides, Kruskal-Wallis test, logistic regression and Wilcoxon rank-sum test were conducted to evaluate the relationship between clinicopathological features and CENPA expression in logistic regression. By pROC package, we 
conducted ROC analysis and common binary assessment for assessing whether CENPA level was effective on separating PRCC cases from healthy subjects. After calculation, AUC values were $0.5-1$, which indicated the $50 \%-100 \%$ discrimination ability. We also collected prognosis data in Cell, and prognostic factors were assessed based on Kaplan-Meier (KM) and Cox regression analyses. $\mathrm{P}<0.05$ stood for statistical significance for each test.

\section{Results}

\subsection{CENPA expression elevated in PRCC samples}

CENPA expression in 289 tumor tissues was substantially higher than that in 32 normal tissues $(\mathrm{P}<$ 0.001) (Fig. 1A), and it was also higher in 31 tumor tissues $(P<0.001)$ (Fig. 1B). Moreover, CENPA expression exhibited promising discrimination ability, with an AUC value of 0.936 in distinguishing tumors from normal tissues (Fig. 1C).

\subsection{Association between CENPA expression and clinicopathological variables}

As revealed by Kruskal-Wallis rank-sum test, CENPA expression was related to pathological T stage ( $\mathrm{P}<$ $0.001)$, pathological $N$ stage $(P<0.001)$, and pathological $M$ stage $(P<0.001)$, clinical stage $(P<0.001)$, and primary treatment effects $(P=0.007)(F i g .2 A-2 E)$. At the same time, results from Wilcoxon rank-sum test indicated that CENPA expression was significantly associated with $\mathrm{T}$ stage, $\mathrm{N}$ stage, $\mathrm{M}$ stage, clinical stage, and primary treatment effect. Furthermore, KM survival analysis demonstrated that high CENPA expression was strongly associated with poor prognosis $(\mathrm{P}<0.001)$ compared with low CENPA expression (Fig. 3A-3B).

\subsection{Role of CENPA up-regulation in predicting PRCC survival under diverse clinicopathological statuses}

For further exploring CENPA-related mechanism within PRCC, this study assessed the relation of CENPA level with clinicopathological features among PRCC cases through univariate Cox regression. More clinicopathological factors related to dismal prognosis included height, weight, primary treatment outcome, pathological T stage, and pathological N stage. For exploring survival-related factors, multivariate Cox regression was conducted on these factors. As a result, CENPA up-regulation was still a factor that independently predicted dismal OS, and other prognostic factors included height, weight, and primary treatment outcome (Table 1). 
Table 1

Association of clinicopathological characteristics with overall survival using univariate or multivariate

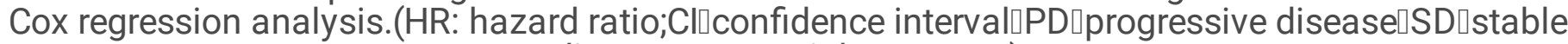
disease $₫ P R \square$ partial response.)

\begin{tabular}{|c|c|c|c|c|c|}
\hline \multirow[t]{2}{*}{ Characteristics } & \multirow[t]{2}{*}{ Total(N) } & \multicolumn{2}{|c|}{ Univariate analysis } & \multicolumn{2}{|l|}{ Multivariate analysis } \\
\hline & & $\begin{array}{l}\text { Hazard } \\
\text { ratio (95\% } \\
\mathrm{Cl})\end{array}$ & $\begin{array}{l}P \\
\text { value }\end{array}$ & Hazard ratio $(95 \% \mathrm{Cl})$ & $\begin{array}{l}P \\
\text { value }\end{array}$ \\
\hline Age & 286 & & & & \\
\hline$<=60$ & 133 & Reference & & & \\
\hline$>60$ & 153 & $\begin{array}{l}0.944 \\
(0.519- \\
1.718)\end{array}$ & 0.851 & & \\
\hline Gender & 288 & & & & \\
\hline Male & 211 & Reference & & & \\
\hline Female & 77 & $\begin{array}{l}1.567 \\
(0.813- \\
3.021)\end{array}$ & 0.180 & & \\
\hline Race & 272 & & & & \\
\hline White & 205 & Reference & & & \\
\hline $\begin{array}{l}\text { Black or } \\
\text { African } \\
\text { American }\end{array}$ & 61 & $\begin{array}{l}0.935 \\
(0.413- \\
2.116)\end{array}$ & 0.871 & & \\
\hline Asian & 6 & $\begin{array}{l}5.966 \\
(0.769- \\
46.311)\end{array}$ & 0.088 & & \\
\hline Height & 212 & & & & \\
\hline$<=170$ & 87 & Reference & & & \\
\hline > 170 & 125 & $\begin{array}{l}0.328 \\
(0.155- \\
0.696)\end{array}$ & 0.004 & $0.000(0.000-0.000)$ & $\begin{array}{l}<.001 \\
0.00\end{array}$ \\
\hline Weight & 218 & & & & \\
\hline$<=80$ & 83 & Reference & & & \\
\hline$>80$ & 135 & $\begin{array}{l}0.413 \\
(0.207- \\
0.827)\end{array}$ & 0.013 & $0.000(0.000-0.000)$ & $\begin{array}{l}<.001 \\
0.00\end{array}$ \\
\hline Laterality & 285 & & & & \\
\hline Left & 159 & Reference & & & \\
\hline
\end{tabular}




\begin{tabular}{|c|c|c|c|c|c|}
\hline \multirow[t]{2}{*}{ Characteristics } & \multirow[t]{2}{*}{ Total(N) } & \multicolumn{2}{|c|}{ Univariate analysis } & \multicolumn{2}{|l|}{ Multivariate analysis } \\
\hline & & $\begin{array}{l}\text { Hazard } \\
\text { ratio }(95 \% \\
\text { Cl) }\end{array}$ & $\begin{array}{l}P \\
\text { value }\end{array}$ & Hazard ratio $(95 \% \mathrm{Cl})$ & $\begin{array}{l}\mathrm{P} \\
\text { value }\end{array}$ \\
\hline Right & 126 & $\begin{array}{l}0.735 \\
(0.392- \\
1.376)\end{array}$ & 0.335 & & \\
\hline $\begin{array}{l}\text { Primary } \\
\text { therapy } \\
\text { outcome }\end{array}$ & 199 & & & & \\
\hline $\mathrm{CR}$ & 185 & Reference & & & \\
\hline PD\&SD\&PR & 14 & $\begin{array}{l}7.157 \\
(3.084- \\
16.612)\end{array}$ & $<001$ & $0.000(0.000-0.001)$ & ¿. 001 \\
\hline $\begin{array}{l}\text { Pathologic T } \\
\text { stage }\end{array}$ & 286 & & & & \\
\hline T1\&T2 & 225 & Reference & & & \\
\hline T3\&T4 & 61 & $\begin{array}{l}5.121 \\
(2.790- \\
9.397)\end{array}$ & $<.001$ & $\begin{array}{l}76417798304913744.000 \\
(7520202477862371.000- \\
776532269571339520.000)\end{array}$ & <. 001 \\
\hline $\begin{array}{l}\text { Pathologic } N \\
\text { stage }\end{array}$ & 77 & & & & \\
\hline NO & 49 & Reference & & & \\
\hline N1\&N2 & 28 & $\begin{array}{l}5.003 \\
(2.062- \\
12.140)\end{array}$ & $\hat{0} 001$ & $0.000(0.000-0.000)$ & $\begin{array}{l}< \\
0.001\end{array}$ \\
\hline Clinical stage & 198 & & & & \\
\hline $\begin{array}{l}\text { Stage I\&Stage } \\
\|\end{array}$ & 159 & Reference & & & \\
\hline $\begin{array}{l}\text { Stage } \\
\text { III\&Stage IV }\end{array}$ & 39 & $\begin{array}{l}9.489 \\
(4.596- \\
19.592)\end{array}$ & $<.001$ & $1.000(0.098-10.162)$ & 1.000 \\
\hline CENPA & 288 & & & & \\
\hline Low & 144 & Reference & & & \\
\hline High & 144 & $\begin{array}{l}3.102 \\
(1.597- \\
6.028)\end{array}$ & $<001$ & $\begin{array}{l}282613493.616(27811723.552- \\
2871824416.970)\end{array}$ & $\begin{array}{l}< \\
0.001\end{array}$ \\
\hline
\end{tabular}

\subsection{Elevated CENPA expression predicts poor prognosis in different cancer stages}


As revealed by $\mathrm{KM}$ method, $\mathrm{PRCC}$ cases in the following tumor stages were associated with dismal survival, including clinical stage (I \& II, $P=0.037$, III \& IV, $P<0.001), T(T 1 \& T 2, P=0.034, T 3 \& T 4, P<$ $0.001), N(N 0, P=0.054, N 1 \& N 2, P=0.003)$, and $M(M 0, P=0.383)$ stages (Fig. 4). Therefore, CENPA level affected PRCC prognosis in diverse clinical stages.

\subsection{Construction and validation of the nomograms based on CENPA expression}

This study established nomograms by integrating survival-related clinicopathological characteristics identified from multivariate analysis (height, weight, CENPA expression level, primary treatment outcome), so as to offer a quantitative approach for clinicians (Fig. 5A). These variables were incorporated into the nomograms based on multivariable Cox analysis using a point scale. We then accumulated the position of the variable and denoted it as a total score. Thereafter, we drew vertical lines from total-point axis along outcome axis to determine the 1-, 3- and 5-year survival probabilities for PRCC cases. After calculation, our nomogram had a C-index value being 0.777 (95\% Cl:0.717-0.837). Moreover, as observed from calibration graph, the deviation alignment line approached the ideal curve (the $45^{\circ}$-line), which indicated that those predicted results were well consistent with actual results (Fig. 5B). On the whole, our nomogram was a superior approach for predicting long-time PRCC prognosis to diverse prognostic factors.

\subsection{Determination of CENPA expression in the samples}

We selected 4 PRCC patients treated in the Urology Department from January 2020 to October 2021, and collected both cancer and normal tissues as CENPA and control groups, respectively. The patient inclusion criteria were presented as follows, (1) patients confirmed with PRCC after paraffin specimen preparation; (2) patients that were diagnosed for the first time; and (3) patients who did not receive radiotherapy, chemotherapy or molecular targeted therapy in our hospital or other hospitals. The current work was approved by the Ethics Committee of our hospital and conducted following Declaration of Helsinki from the World Medical Association. All patients and their families signed the informed consents. The 4-um paraffin sections were washed by xylene and hydrated with gradient ethanol solution to water for deparaffinage and hydration. Later, the sections were boiled within sodium citrate buffer $(0.01 \mathrm{~mol} / \mathrm{L}, \mathrm{pH} 6.0)$ to achieve antigen heat repair for a 20 -min period. Thereafter, $3 \%$ hydrogen peroxide was added dropwise for inactivating tissue endogenous peroxidase. Then, sections were incubated at room temperature for $10 \mathrm{~min}$, washed by $1 \%$ bovine serum albumin (BSA), and incubated for a 30 -min period under ambient temperature. After discarding the blocking solution, sections were incubated with primary antibody (1:100 dilution of CENPA antibody) at $4{ }^{\circ} \mathrm{C}$ overnight, washed with biotin-conjugated secondary antibody for a 40 -min period under $37^{\circ} \mathrm{C}$, and washed with HRP-labeled streptomycin working solution. Afterwards, sections were stained with $D A B$ and hematoxylin, dehydrated and transparentized with gradient ethanol solution and xylene, and sealed with neutral gum. Finally, photographs were taken under an optical microscope. 
Immunohistochemical (IHC) staining was positive for the appearance of blue-purple particles. The score was rated in accordance with the percentage of positive cells and staining intensity. The scores of positive cell percentage were shown below, 0 (<5\%), 1 (5\% 25\%), 2 (26\% 50\%), 3 (51-75\%), and 4 (> $75 \%$ ), while the scores of staining intensity were as follows, 0 (no color or unclear), 1 (light purple), 2 (blue purple), and 3 (dark purple). Thereafter, the staining index (SI) was calculated as the product of positive cell percentage score and staining intensity score, which was expressed as negative for $\mathrm{SI}<2$ and positive for $\mathrm{SI}>2$. IHC results showed that the CENPA-positive rate was lower in control group than in PRCC group (Fig. 6A-6B).

\subsection{Identification of DEGs between high and low CENPA expression groups}

We utilized the R software DSEeq2 package to analyze TCGA-derived data (adjusted $\mathrm{P}<0.05$ and $|\log \mathrm{FC}|>1.5)$ and discovered altogether 1210 DEGs between CENPA up-regulation and down-regulation groups, among which, 1170 showed up-regulation while 40 showed down-regulation in CENPA upregulation group (Fig. 7A-7B).

\subsection{Functional annotation and prediction of signaling pathways}

For further understanding CENPA's role in PRCC prognosis based on those 1,210 DEGs discovered between CENPA up-regulation and down-regulation groups, we carried out $\mathrm{GO}$ annotation by ClusterProfile package. We obtained 233 GO-biological process (GO-BP) terms, such as mitosis, chromosome segregation, and nuclear division. (Fig. 8A). The above findings indicated that abnormal CENPA level was related to nuclear division. In the meantime, we also discovered $18 \mathrm{GO}$-cellular component (GO-CC) terms, which confirmed that the abnormal CENPA expression was associated with centrotochore (Fig. 8B). In addition, the GO-molecular function (GO-MF) terms identified included the transcription to DNA-binding activator activity, typically, the RNA polymerase II was significantly enriched (Fig. 8C).

\subsection{The CENPA-associated signaling pathway based on GSEA}

Subsequently, signaling pathways related to PRCC were identified between high and low CENPA expression groups through GSEA using significant difference in MSigDB Collection (c2.all.v7.0) (adjusted $P<0.05$, FDR $<0.25$ ). Finally, 5 pathways, including KEGG - NEUROACTIVE - LIGAND - RECEPTOR INTERACTION, KEGG - CYTOKINE - CYTOKINE - RECEPTOR INTERACTION, NABA - ECM - REGULATORS, NABA - ECM - GLYCOPROTEINS, and NABA - CORE - MATRISOME pathways, were identified with significant differences between both groups (Fig. 9A-E). 


\subsection{Correlation between CENPA expression and immune infiltration}

At last, this study examined the relation of CENPA level (TPM) with the enrichment levels of immune cells (obtained from sSGSEA) according to Spearman correlation analysis. As a result, CENPA level showed negative relation to enrichment levels of B cells, Cytotoxic cells, Nrutrophlis, NK CD56bright cells, CD8 T cells, TEM, iDC, Macrophages, DC, Mast cells, and Eosinophils. Moreover, CENPA level was positively associated with enrichment levels of Tcm, T cells, NK cells, Th17 cells, TReg, Th1 cells, TFH, T CD56dim cells, pDC, aDC, Th2 cells and Tgd (Fig. 10).

\section{Discussion}

PRCC is the second most common type of renal cancer, however, there are few molecular mechanism studies and randomized clinical trials on PRCC due to the limited number of cases ${ }^{[3]}$. Recently, due to the rapid development of bioinformatics, the molecular mechanisms related to PRCC progression are being continuously explored. Although there are many clinical methods to treat PRCC, the patient prognosis remains poor. Therefore, it is of great necessity to identify the effective prognostic biomarkers to accurately predict PRCC prognosis and improve patient survival. With the continuous maturity of RNA sequencing and gene chip technology, the accuracy has been greatly improved, and the cancer-related big data network has been formed. In this regard, bioinformatics-based big data mining can easily find genes possibly related to the occurrence and development of PRCC. At present, massive studies are being conducted based on the bioinformatics prognostic analysis of PRCC ${ }^{[4-8]}$.

The CENP family is considered as an important functional gene related to tumor development. High expression of centromere protein $\mathrm{H}(\mathrm{CENPH})$ is suggested to predict the poor prognosis of patients with tongue cancer ${ }^{[9]}$, whereas overexpression of kinetocentromeric protein M (CENPM) can promote the occurrence of liver cancer ${ }^{[10]}$, and overexpression of kinetocentromeric protein $\mathrm{F}$ (CENPF) can promote the development of NPC, GISTs and breast cancer $(B C)^{[11]}$. CENPA in this study is a major component of the CENP family. As a centromere-specific variant, CENPA is identified as a key epigenetic marker for centromere recognition and reproduction, which has two main functions, including (1) providing essential conditions for centromere formation and maintenance, and (2) forming a platform for centromere assembly and mediating chromosome isolation ${ }^{[12]}$. This study again demonstrated these points by performing gene enrichment analysis on PRCC patient samples. As a result, abnormally high or low CENPA expression might disrupt genome integrity, resulting in chromosomal mis-polymerization and tumor occurrence. Previous studies have found that CENPA is highly expressed in more than 20 cancer tissues, including BC, colorectal cancer (CRC), lung adenocarcinoma (LUAD) and ovarian cancer ${ }^{[13-16]}$. Nonetheless, the effect of CENPA on predicting PRCC prognosis remains largely unclear. According to this study, CENPA served as the creditable factor to predict PRCC survival. 
This work conducted bioinformatics analysis based on TCGA-derived high-throughput RNA-seq data for assessing CENPA's effect on predicting PRCC survival. We found that CENPA expression within tumor tissue was higher than that in normal samples. Meanwhile, studies have discovered that overexpression of CENPA in cancer tissues is associated with poor clinicopathological factors, implying that CENPA most likely acts as an oncogene. Alternatively, high CENPA level was markedly related to dismal OS compared with low CENPA expression. Therefore, we speculated that CENPA was the biomarker for PRCC. For better investigating CENPA's effect on PRCC, the TCGA-derived data based on GSEA were utilized. As a result, associated genes regulated by cytokine receptor interactions, neuroactive ligand receptor interactions, nuclear mother, extracellular matrix glycoproteins, and extracellular matrix regulators were differentially enriched in the PRCC phenotype with high CENPA expression. However, whether CENPA exerts the synergistic or complementary effects with these pathways remains unclear, and detailed regulatory networks are unavailable for the time being. Moreover, CENPA was related to diverse complicated crosswalk related to additional genes. Consequently, mechanisms related to the above interactions should be further investigated in details.

Tumor microenvironment (TME) has been considered as an important interface that mediates tumor cells' physiological responses, while tumor-infiltrating immune cells (TIICs) represent a crucial component in TME, and their levels and composition have been identified to be related to tumor survival ${ }^{[17-18]}$. On this basis, this work analyzed the relation of CENPA level with immune infiltration degrees within PRCC (Fig. 9). It suggested that the increased CENPA expression was inversely related to the abundance levels of B cells, CD8 + T cells and Treg in PRCC. Besides, it has been shown that the low levels of B cells and $\mathrm{CD} 8+\mathrm{T}$ cells in tumor tissues are associated with the prognosis of cancer patients ${ }^{[19-21]}$, containing the poor prognosis of PRCC. Several other studies report that the high levels of tumor-infiltrating Tregs in BC, $\mathrm{HCC}$, lung cancer, gastric cancer (GC), and ovarian cancer were significantly associated with the poorer prognosis ${ }^{[22-26]}$.Therefore, we reasoned that CENPA might affect patient prognosis by modulating the immune infiltration in PRCC.

Our study provides new insights into the correlation between CENPA and PRCC, but there are some limitations to be noted. First, we only evaluated one TCGA dataset, which might cause sample bias. Secondly, the sample size should be further expanded to increase the credibility of our results. Thirdly, further experimental validation is warranted to elucidate the in-vitro and in-vitro biological functions of CENPA.

\section{Conclusion}

This study reveals for the first time the prognostic value of CENPA in PRCC. Our results suggest that CENPA has the potential as a biomarker to predict treatment outcome and prognosis of PRCC patients. However, further experiments are still needed to validate the biological effects and underlying mechanisms of CENPA. 


\section{Abbreviations}

CENPA Centromeric protein A

PRCC Papillary renal cell carcinoma

ROC Receiver operating feature curve

AUC Value of area under curve

KM Kaplan-Meier

IHC Immunohistochemistry

DEGs Differentially expressed genes

RNA-seq RNA-sequencing

OS Overall survival

PPI Protein-protein interaction

GO Gene Ontology

FDR False discovery rate

ssGSEA Single sample of GSEA

NK cell Natural killer cell

aDC Activated DC

DC Dendritic cell

iDC Immature DC

pDC Plasmacyte-like DC

Th cell $\mathrm{T}$ helper cell

Treg Regulatory $T$ cell

Tem Effector memory T cell

Tcm Central memory T cell

Tgd T cell 
C-index Concordance index

$\mathrm{Cl}$ Confidence interval

BSA Bovine serum albumin

SI Staining index

BP Biological process

CC Cellular component

MF Molecular function

CENPH Centromere protein $\mathrm{H}$

CENPM Kinetocentromeric protein M

CENPF Kinetocentromeric protein $\mathrm{F}$

BC Breast cancer

NPC Nasopharyngeal carcinoma

GISTs Gastrointestinal stromal tumors

CRC Colorectal cancer

LUAD Lung adenocarcinoma

TME Tumor microenvironment

TIICs Tumor-infiltrating immune cells

HCC Hepatocellular-cancer

GC Gastric cancer

\section{Declarations}

\section{Ethics approval and consent to participate}

This trial was conducted in accordance with the principles of the Declaration of Helsinki and Good Clinical Practice guidelines. The study protocol was approved by the ethics committee of the Second Affiliated Hospital of Chongqing Medical University (Chongqing, China) and regulatory authorities. 
Not applicable.

\section{Availability of data and material}

The material supporting the conclusion of this review has been included within the article.

\section{Competing interests}

The authors declare that they have no competing interests.

\section{Funding}

This work was supported by the Research Program of Natural Science Foundation in Chongqing (cstc2021jcyj-msxmX0484), National Natural Science Foundation of China(No.81801507) and Kuanren Talent Program of Second Affiliated Hospital of Chongqing Medical University (KY2019Y004).

\section{Authors' contributions}

JL, $Y Z$ and RZ designed the study. JL, QL and $Y Z$ drafted the manuscript. JL and $Y X$ prepared the table and figure. JL, QL and YZ collected specimens and completed the immunohistochemistry. All authors participated in the revision of the manuscript. All authors read and approved the final manuscript.

\section{Acknowledgments}

Not applicable.

\section{Contributor Information}

Junwu Li: 2020120886@stu.cqmu.edu.cn

Qinke Li: Iqkcqmu@126.com

Yiteng Xie: xyt11223345@163.com

Yuanfeng Zhang: 304100@cqmu.edu.cn

Ronggui Zhang: 300452@hospital.cqmu.edu.cn

\section{References}

1. Courthod G, Tucci M, Di Maio M, et al. Papillary renal cell carcinoma: A review of the current therapeutic landscape. Crit Rev Oncol Hematol. 2015. 96(1): 100-12.

2. Akhtar M, Al-Bozom IA, Al Hussain T. Papillary Renal Cell Carcinoma (PRCC): An Update. Adv Anat Pathol. 2019. 26(2): 124-132. 
3. Sun Z, Jing C, Xiao C, et al. Prognostic risk signature based on the expression of three m6A RNA methylation regulatory genes in kidney renal papillary cell carcinoma. Aging (Albany NY). 2020. 12(21): 22078-22094.

4. Cui JH, Zhao M, Niu HT, et al. Bioinformatics analysis of differentially expressed genes and pathways in papillary renal cell carcinoma. Journal of Qingdao University (Medical Edition). 2019. 55(04): 402-405 + 410 .

5. Zheng HH, Wen SX, Gan FM. Expression and significance of GATA3 in clear cell papillary renal cell carcinoma. Journal of Practical Oncology. 2020. 35(02): 150-153.

6. Li QJ, Baihetiya A, Wang WG, et al. Analysis of clinical features and prognostic factors in patients with papillary renal cell carcinoma. Journal of Xinjiang Medical University. 2020. 43(05): 577-581.

7. Gu H. Expression and clinical significance of GTSE1 gene in papillary renal cell carcinoma. Journal of Modern Genitourinary Oncology. 2020. 12(03): 153-156.

8. Liu DY, Zhang S, Xu YK, et al. Independent prognostic role of TERT gene in papillary renal cell carcinoma. Journal of China Medical University. 2021. 50(07): 616-620.

9. Liao WT, Yu CP, Wu DH, et al. Upregulation of CENP-H in tongue cancer correlates with poor prognosis and progression. Exp Clin Cancer Res. 2009. 28(1): 74.

10. Xiao Y, Najeeb RM, Ma D, et al. Upregulation of CENPM promotes hepatocarcinogenesis through mutiple mechanisms. Exp Clin Cancer Res. 2019. 38(1): 458.

11. Sun J, Huang J, Lan J, et al. Overexpression of CENPF correlates with poor prognosis and tumor bone metastasis in breast cancer. Cancer Cell Int. 2019. 19: 264.

12. Tomonaga $\mathrm{T}$, Matsushita $\mathrm{K}$, Yamaguchi $\mathrm{S}$, et al. Overexpression and mistargeting of centromere protein-A in human primary colorectal cancer. Cancer Res. 2003. 63(13): 3511-6.

13. Rajput $A B, H u N$, Varma S, et al. Immunohistochemical Assessment of Expression of Centromere Protein-A (CENPA) in Human Invasive Breast Cancer. Cancers (Basel). 2011. 3(4): 4212-27.

14. Qiu JJ, Guo JJ, Lv TJ, et al. Prognostic value of centromere protein-A expression in patients with epithelial ovarian cancer. Tumour Biol. 2013. 34(5): 2971-5.

15. Wu Q, Qian YM, Zhao XL, et al. Expression and prognostic significance of centromere protein $A$ in human lung adenocarcinoma. Lung Cancer. 2012. 77(2): 407-14.

16. Gu XM, Fu J, Feng XJ, et al. Expression and prognostic relevance of centromere protein A in primary osteosarcoma. Pathol Res Pract. 2014. 210(4): 228-33.

17. Nguyen $N$, Bellile $E$, Thomas $D$, et al. Tumor infiltrating lymphocytes and survival in patients with head and neck squamous cell carcinoma. Head Neck. 2016. 38(7): 1074-84.

18. Wolf GT, Chepeha DB, Bellile E, et al. Tumor infiltrating lymphocytes (TIL) and prognosis in oral cavity squamous carcinoma: a preliminary study. Oral Oncol. 2015. 51(1): 90-5.

19. Sandoval F, Terme M, Nizard M, et al. Mucosal imprinting of vaccine-induced CD8âo T cells is crucial to inhibit the growth of mucosal tumors. Sci Transl Med. 2013. 5(172): 172ra20. 
20. Fousek K, Ahmed N. The Evolution of T-cell Therapies for Solid Malignancies. Clin Cancer Res. 2015. 21(15): 3384-92.

21. Peng YL, Xiong LB, Zhou ZH, et al. Single-cell transcriptomics reveals a low CD8(+) T cell infiltrating state mediated by fibroblasts in recurrent renal cell carcinoma. Immunother Cancer. 2022. 10(2).

22. Bates GJ, Fox SB, Han C, et al. Quantification of regulatory $T$ cells enables the identification of highrisk breast cancer patients and those at risk of late relapse. Clin Oncol. 2006. 24(34): 5373-80.

23. Wang Y, Liu T, Tang W, et al. Hepatocellular Carcinoma Cells Induce Regulatory T Cells and Lead to Poor Prognosis via Production of Transforming Growth Factor- $\beta 1$. Cell Physiol Biochem. 2016. 38(1): 306-18.

24. Petersen RP, Campa MJ, Sperlazza J, et al. Tumor infiltrating Foxp3 + regulatory T-cells are associated with recurrence in pathologic stage I NSCLC patients. Cancer. 2006. 107(12): 2866-72.

25. Hu M, Li K, Maskey N, et al. Decreased intratumoral Foxp3 Tregs and increased dendritic cell density by neoadjuvant chemotherapy associated with favorable prognosis in advanced gastric cancer. Int $\mathrm{J}$ Clin Exp Pathol. 2014. 7(8): 4685-94.

26. Curiel TJ, Coukos G, Zou L, et al. Specific recruitment of regulatory $T$ cells in ovarian carcinoma fosters immune privilege and predicts reduced survival. Nat Med. 2004. 10(9): 942-9.

\section{Tables}

Table 1 Association of clinicopathological characteristics with overall survival using univariate or

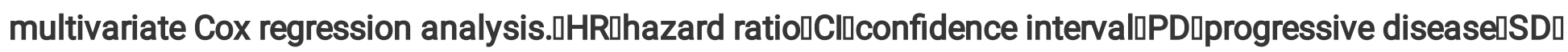
stable disease $\triangle P R \square$ partial response. $\square$ 
Characteristics Total(N) Univariate analysis Multivariate analysis

$\begin{array}{llll}\text { Hazard } & \mathrm{P} & \text { Hazard ratio }(95 \% \mathrm{Cl}) & \mathrm{P} \\ \text { ratio }(95 \% & \text { value } & & \text { value } \\ \mathrm{Cl}) & & \end{array}$

\begin{tabular}{|c|c|c|c|c|c|}
\hline Age & 286 & & & & \\
\hline$<=60$ & 133 & Reference & & & \\
\hline$>60$ & 153 & $\begin{array}{l}0.944 \\
(0.519- \\
1.718)\end{array}$ & 0.851 & & \\
\hline Gender & 288 & & & & \\
\hline Male & 211 & Reference & & & \\
\hline Female & 77 & $\begin{array}{l}1.567 \\
(0.813- \\
3.021)\end{array}$ & 0.180 & & \\
\hline Race & 272 & & & & \\
\hline White & 205 & Reference & & & \\
\hline $\begin{array}{l}\text { Black or } \\
\text { African } \\
\text { American }\end{array}$ & 61 & $\begin{array}{l}0.935 \\
(0.413- \\
2.116)\end{array}$ & 0.871 & & \\
\hline Asian & 6 & $\begin{array}{l}5.966 \\
(0.769- \\
46.311)\end{array}$ & 0.088 & & \\
\hline Height & 212 & & & & \\
\hline$<=170$ & 87 & Reference & & & \\
\hline$>170$ & 125 & $\begin{array}{l}0.328 \\
(0.155- \\
0.696)\end{array}$ & 0.004 & $0.000(0.000-0.000)$ & $<0.001$ \\
\hline Weight & 218 & & & & \\
\hline$<=80$ & 83 & Reference & & & \\
\hline$>80$ & 135 & $\begin{array}{l}0.413 \\
(0.207- \\
0.827)\end{array}$ & 0.013 & $0.000(0.000-0.000)$ & $<0.001$ \\
\hline Laterality & 285 & & & & \\
\hline Left & 159 & Reference & & & \\
\hline Right & 126 & $\begin{array}{l}0.735 \\
(0.392- \\
1.376)\end{array}$ & 0.335 & & \\
\hline
\end{tabular}


Characteristics Total(N) Univariate analysis Multivariate analysis

$\begin{array}{llll}\text { Hazard } & \mathrm{P} & \text { Hazard ratio }(95 \% \mathrm{Cl}) & \mathrm{P} \\ \text { ratio }(95 \% & \text { value } & & \text { value } \\ \mathrm{Cl}) & & \end{array}$

Primary

therapy

outcome
199

\begin{tabular}{llllll} 
CR & 185 & \multicolumn{2}{l}{ Reference } & & \\
PD\&SD\&PR & 14 & $\begin{array}{l}7.157 \\
(3.084-\end{array}$ & $<0.001$ & $0.000(0.000-0.001)$ & $<0.001$ \\
& & & & \\
& & &
\end{tabular}

Pathologic T 286
stage

\begin{tabular}{llllll} 
T1\&T2 & 225 & Reference & & & \\
T3\&T4 & 61 & 5.121 & $<0.001$ & 76417798304913744.000 & $<0.001$ \\
& & $(2.790-$ & & $(7520202477862371.000-$ & \\
& $9.397)$ & & $776532269571339520.000)$ & \\
\hline
\end{tabular}

Pathologic N 77

stage

$\begin{array}{llllll}\text { N0 } & 49 & \text { Reference } & & & \\ \text { N1\&N2 } & 28 & 5.003 & <0.001 & 0.000(0.000-0.000) & <0.001 \\ & & (2.062- & & & \\ & & & \end{array}$

Clinical stage $\quad 198$

Stage I\&Stage $159 \quad$ Reference

II

$\begin{array}{lllll}\text { Stage } & 39 & 9.489 & <0.001 & 1.000(0.098-10.162) \\ \text { III\&Stage IV } & & (4.596- & & 1.000 \\ & & & \end{array}$

CENPA 288

$\begin{array}{llllll}\text { Low } & 144 & \text { Reference } & & \\ \text { High } & 144 & \begin{array}{l}3.102 \\ (1.597-\end{array} & <0.001 & 282613493.616(27811723.552- & <0.001 \\ & & 6.028) & & 2871824416.970) & \\ & & & \end{array}$

\section{Figures}




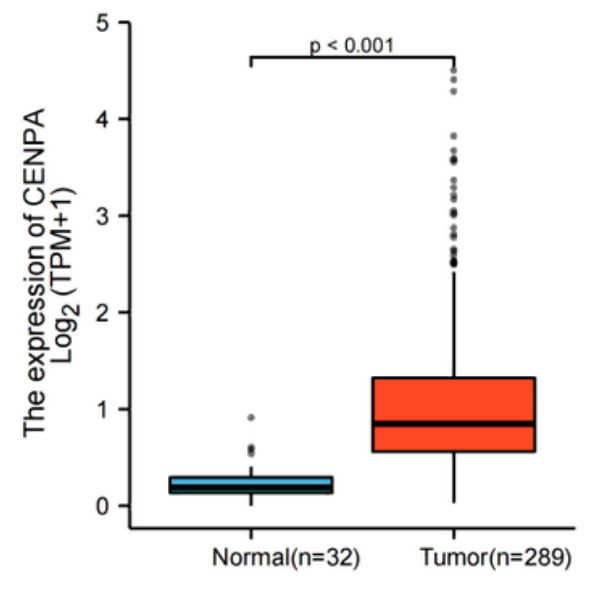

$(1 \mathrm{~A})$

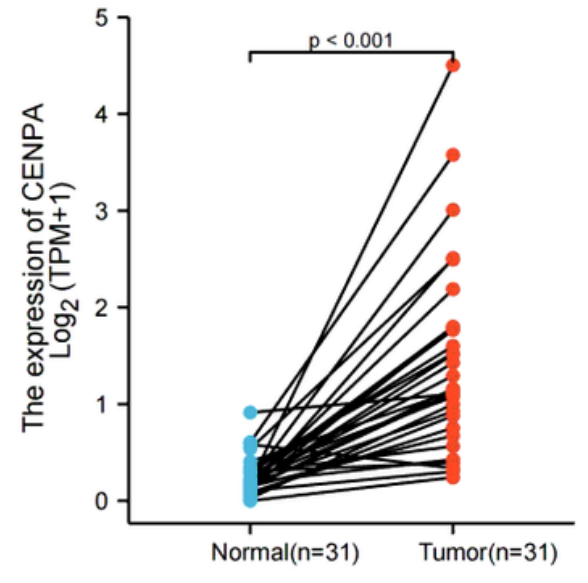

(1B)

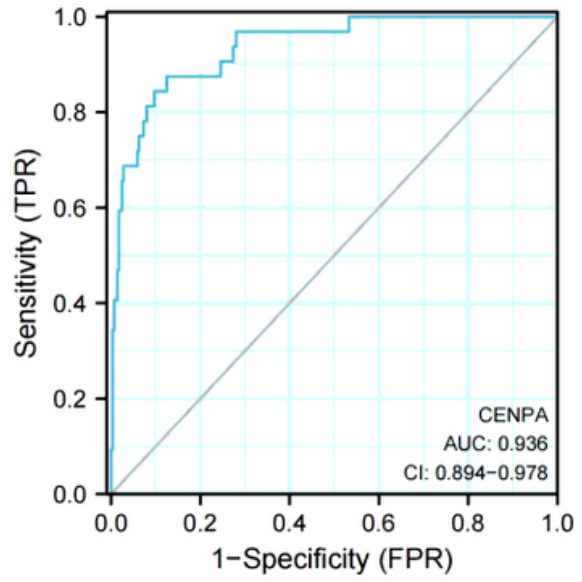

(1C)

\section{Figure 1}

CENPA expression between cancer and normal tissues in PRCC patients. CENPA expression levels in PRCC and matched normal tissues $\triangle 1 A \bigotimes$. CENPA expression levels in PRCC and marched normal tissues $\triangle 1 \mathrm{~B} \bigotimes$. ROC analysis of CENPA shows promising discrimination power between tumor and normal tissue Q1C】. 


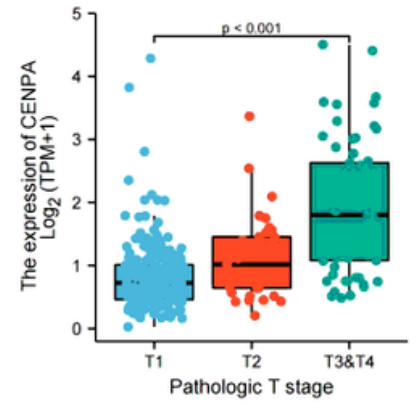

(2A)

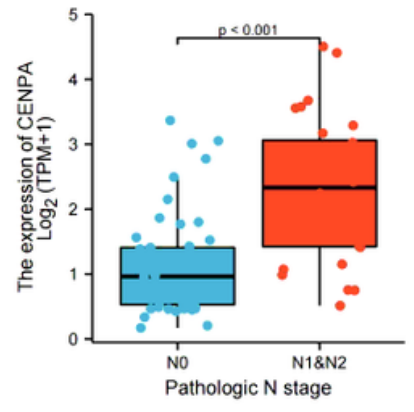

(2B)

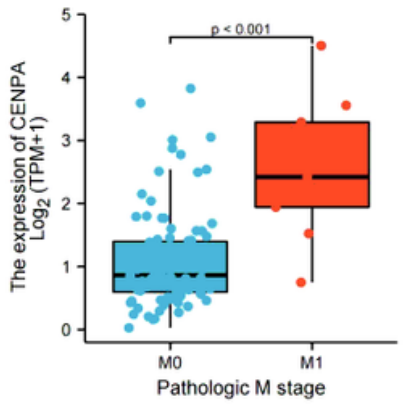

(2C)

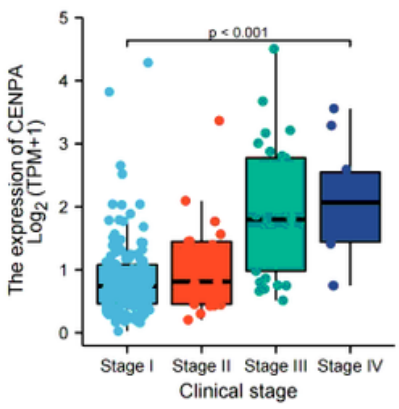

(2D)

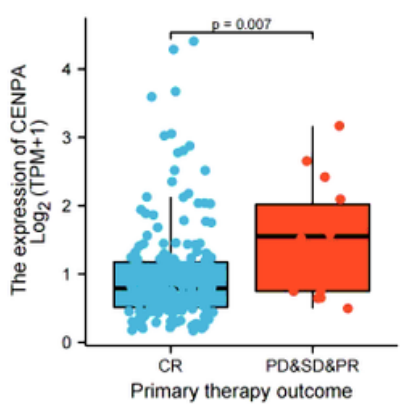

(2E)

\section{Figure 2}

Association of CENPA expression with clinicopathologic characteristics. Pathologic T stage $\mathbb{2 A} \mathrm{A} \otimes$

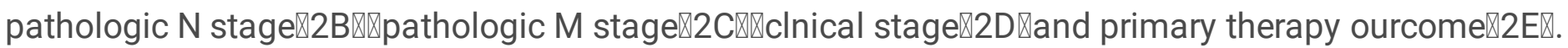




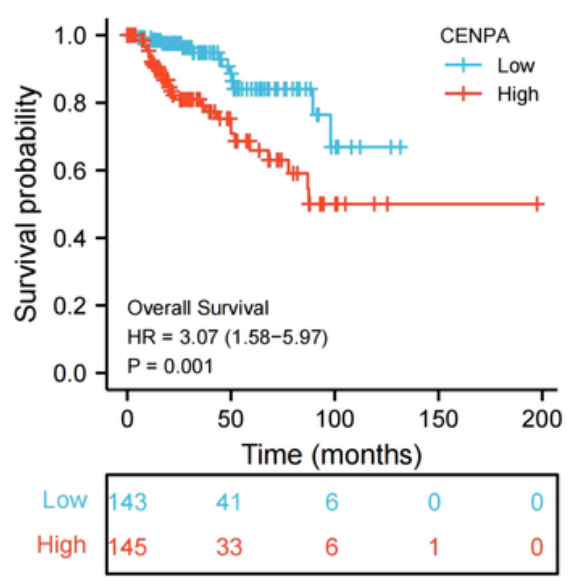

$(3 A)$

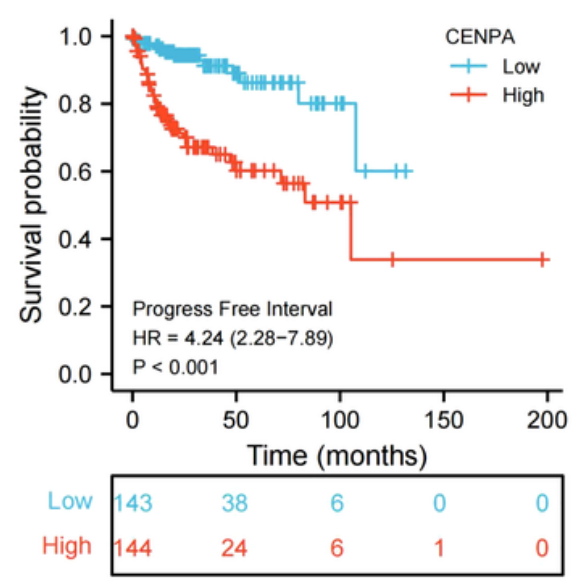

(3B)

Figure 3

Kaplan-Meier survival curves comparing the high and low expression of CENPA in PRCC patients. Overall

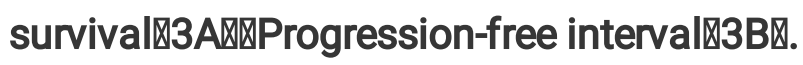

\begin{tabular}{|c|c|c|c|c|}
\hline Characteristics & N (\%) & HR (95\% Cl) & & $P$ value \\
\hline T stage & & & 1 & \\
\hline T1\&T2 & $226(78.7 \%)$ & 2.68(1.14-6.30) & & 0.034 \\
\hline T3\&T4 & $63(21.3 \%)$ & $8.00(3.26-19.65$ & 1 & $<0.001$ \\
\hline $\mathrm{N}$ stage & & & 1 & \\
\hline No & $49(63.6 \%)$ & $4.29(0.92-19.94)$ & $\longrightarrow$ & 0.054 \\
\hline N1\&N2 & $28(36.4 \%)$ & $3.69(1.35-10.14)$ & -1 & 0.003 \\
\hline M stage & & & I & \\
\hline MO & $95(91.3 \%)$ & $1.62(0.55-4.82)$ & $\mathrm{H} \longrightarrow \mathrm{C}$ & 0.383 \\
\hline Clinical stage & & & I & \\
\hline Stage I\&II & $159(80.3 \%)$ & $3.63(1.17-11.32)$ & & 0.037 \\
\hline Stage III\&IV & $39(19.7 \%)$ & 7.17(2.64-19.50) & I & $<0.001$ \\
\hline
\end{tabular}


Figure 4

Multivariate survival analysis of overall survival probabilities concerning CENPA expression in patients of different subgroups according to cancer stage.

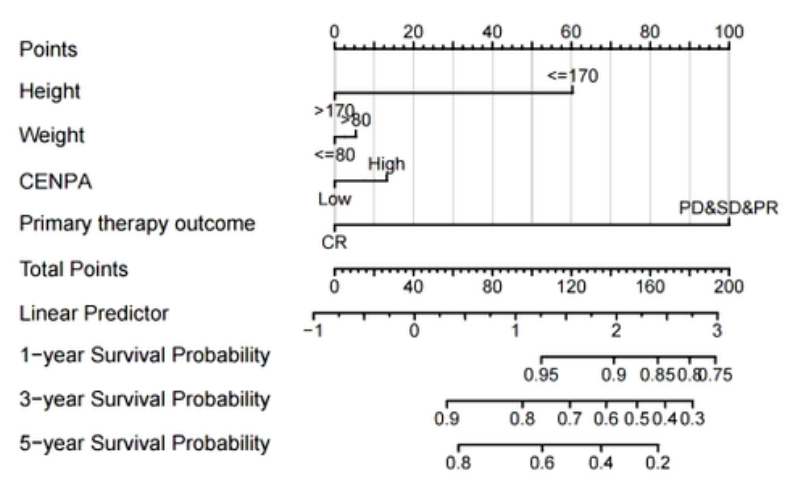

$(5 \mathrm{~A})$

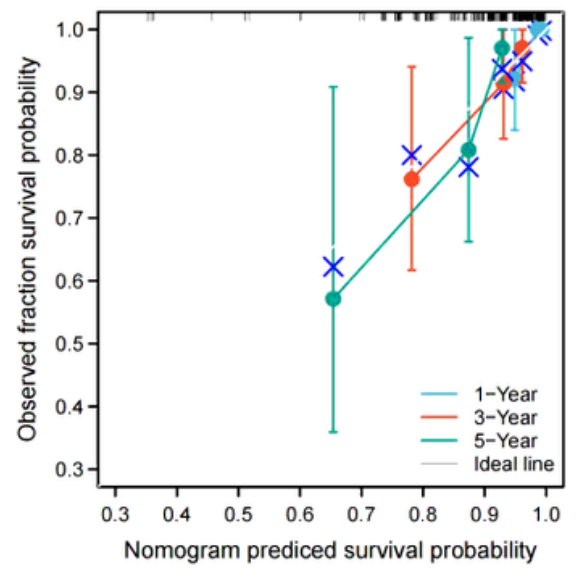

(5B)

Figure 5

Relationship between CENPA and other clinical factors with overall survivalखOS区. Nomogram for

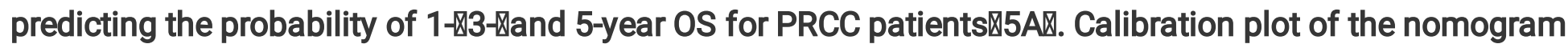
for predicting the OS likelihood $₫ 5 \mathrm{~B}$. 

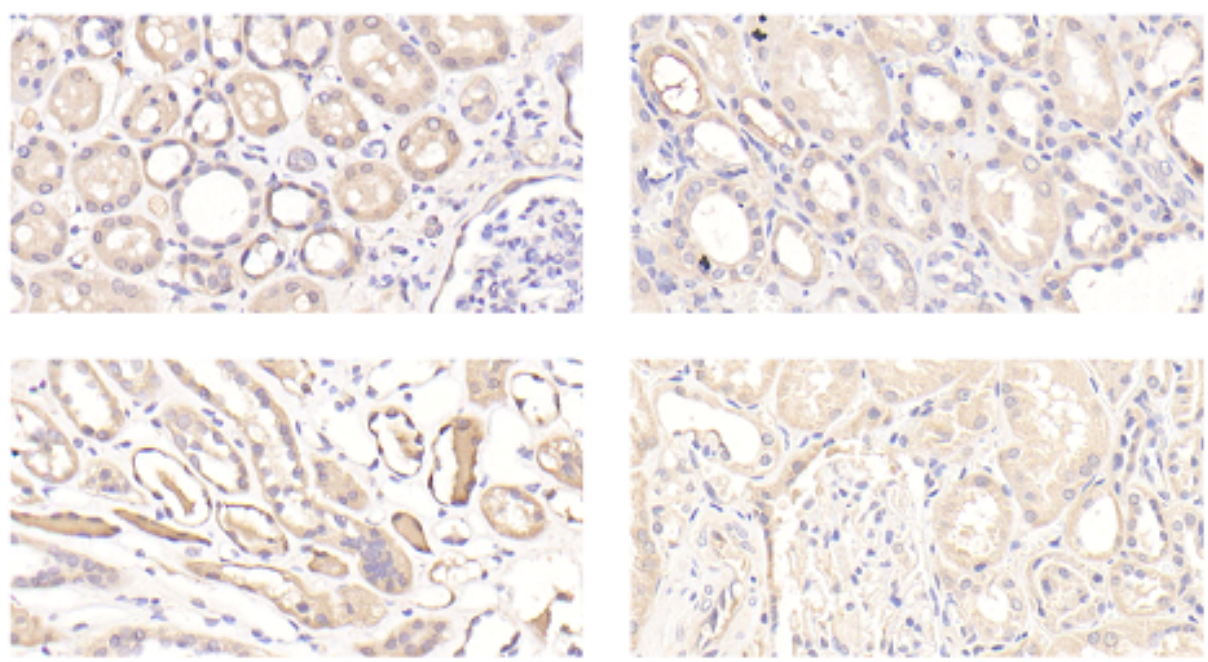

(6A)
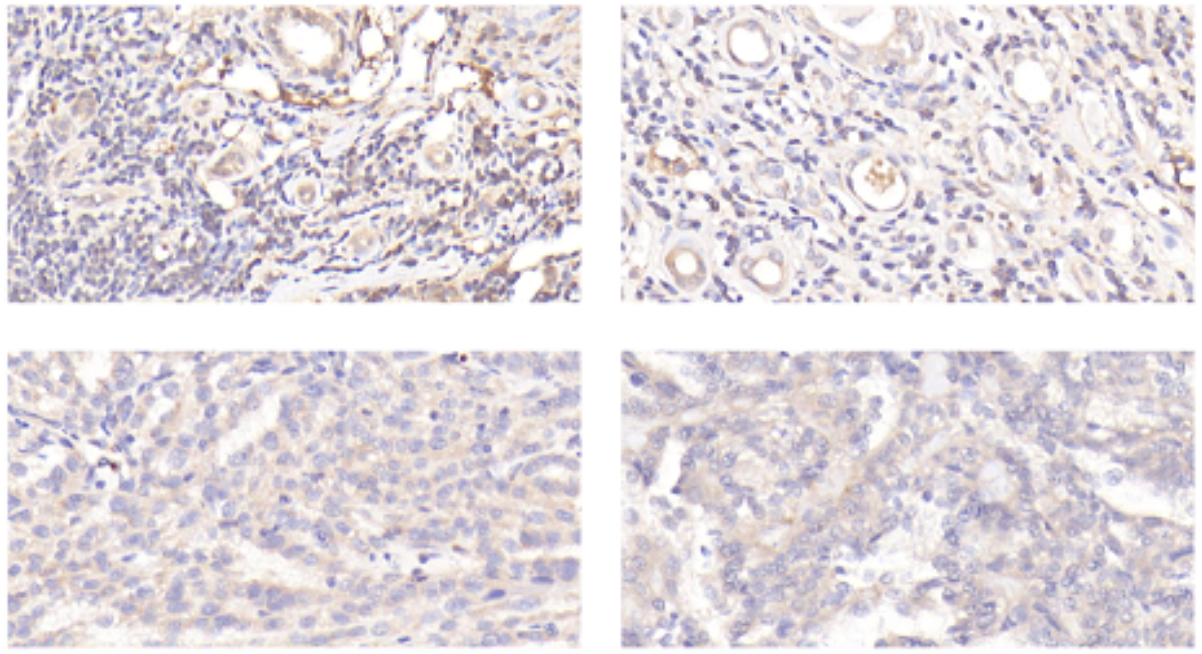

(6B)

Figure 6

Expression of CENPA in the two gruops detected by immunohistochemistry staining(x400)(6A:Control group;6B:PRCC group) 


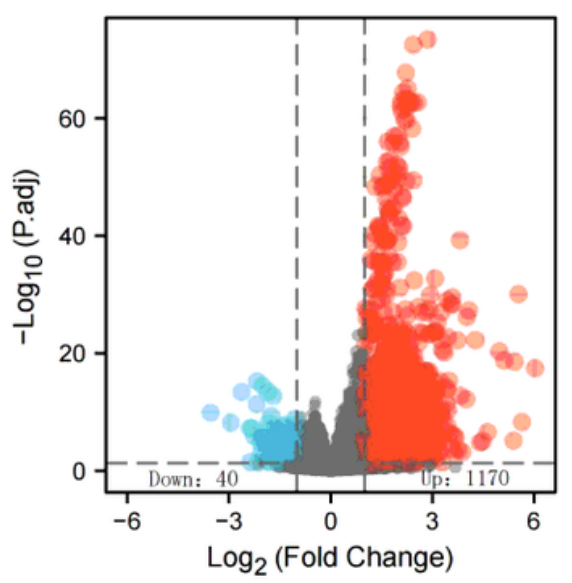

$(7 \mathrm{~A})$

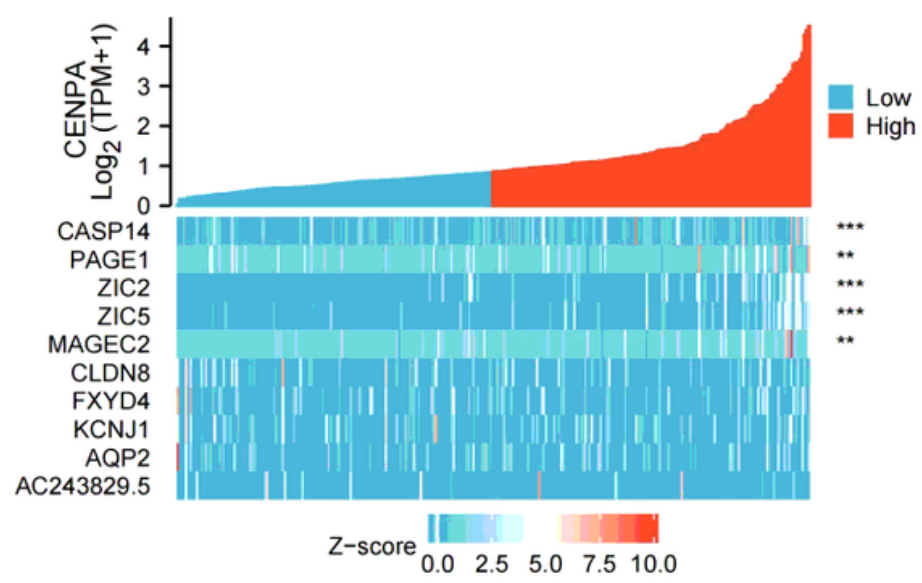

(7B)

Figure 7

Differentially expressed genes between patients with high and low CENPA expression. Volcano plot of differentially expressed genes between the high and low CENPA expression groups. Normalized expression levels are shown in descending order from blue to redX7AX. Heatmap of the top ten significant differentially expressed genes between the high and low CENPA expression groups. Blue and red dots represent downregulated and upregulated genes囚respectively囚7B囚. 


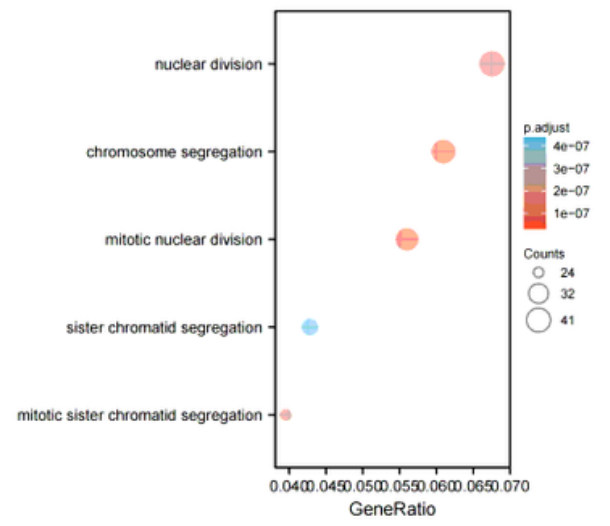

$(8 \mathrm{~A})$

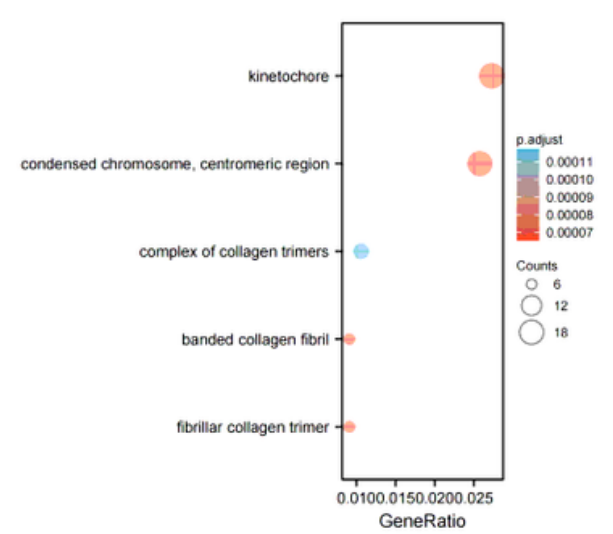

(8B)

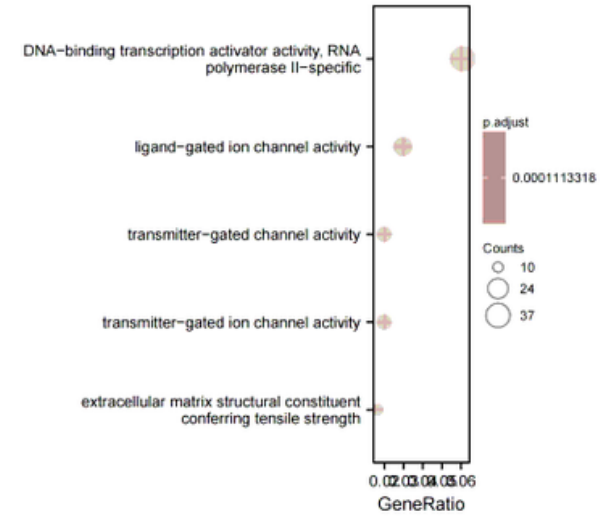

$(8 \mathrm{C})$

Figure 8

Enriched GO terms in the "biological process" category $\$ 8$ AD. Enriched GO terms in the "cellular component" category $₫ 8 \mathrm{~B} \bigotimes$. Enriched GO terms in the "molecular function" category $₫ 8 \mathrm{C} \Downarrow$. The $\mathrm{x}$-axis represents the proportion of differentially expressed genes囚DEGs囚and the $y$-axis represents different categories. Blue and red tones represent adjusted $\mathrm{P}$ values form 0.0 to 0.05》respectively囚and different circle sizes represent the number of DEGs. 


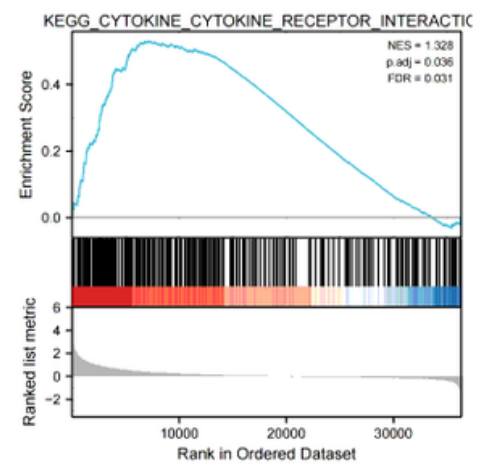

(9A)

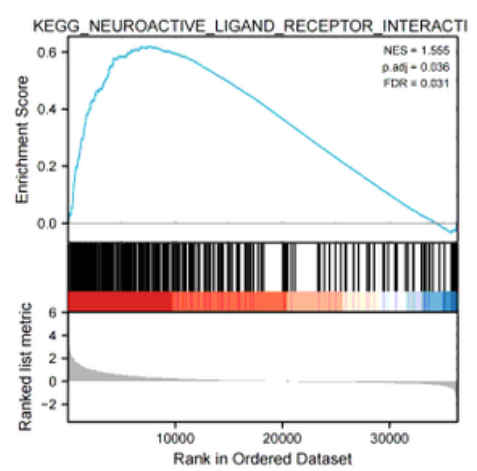

(9B)

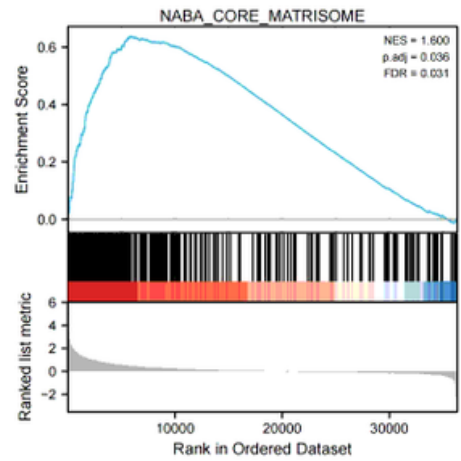

(9C)

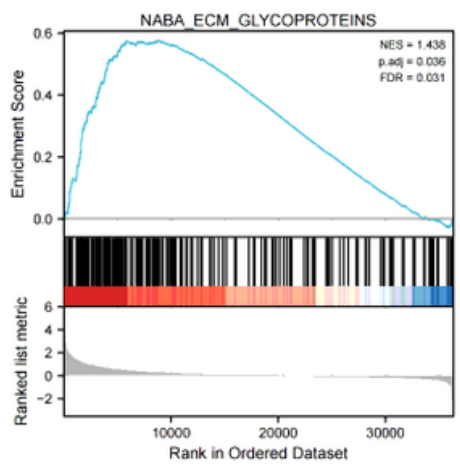

(9D)

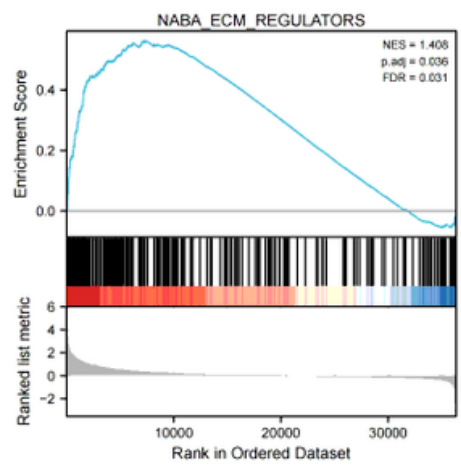

(9E)

Figure 9

Enrichment plots from GESA. Several pathway were differentially enriched in PRCC patients according to high and low CENPA expression. KEGG - CYTOKINE - CYTOKINE - RECEPTOR - INTERACTION pathway

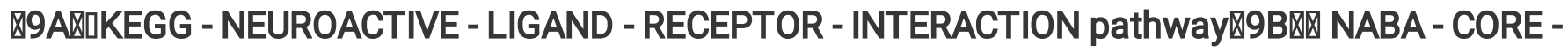

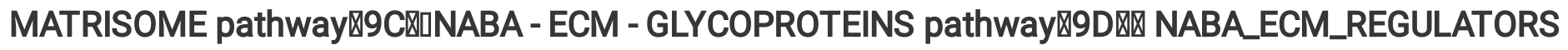

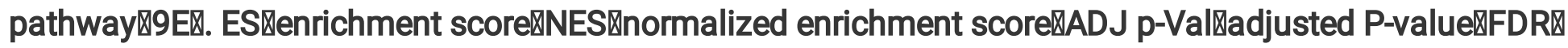
false discovery rate. 


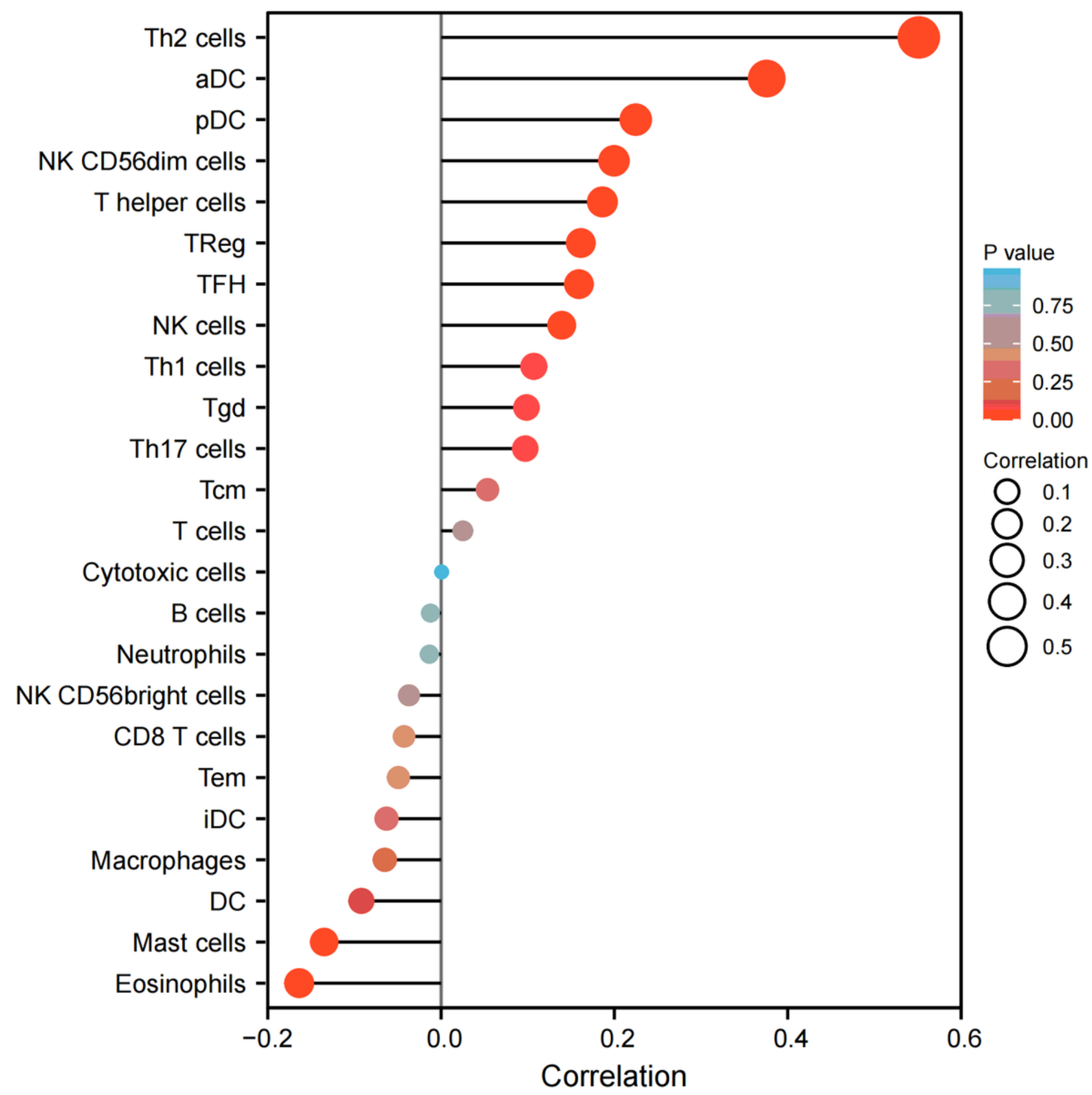

Figure 10

Correlations between the relative abundance of 24 immune cells and CENPA expression levels. The size of the dots represents the absolute Spearman's correlation coefficient values. 